Research Article

\title{
Track-to-Track Association Based on Structural Similarity in the Presence of Sensor Biases
}

\author{
Hongyan Zhu and Suying Han \\ School of Electronic and Information Engineering, Xian Jiaotong University, Xian 710049, China \\ Correspondence should be addressed to Hongyan Zhu; hyzhu2011@gmail.com
}

Received 23 October 2013; Accepted 26 February 2014; Published 25 March 2014

Academic Editor: Nachamada Blamah

Copyright (C) 2014 H. Zhu and S. Han. This is an open access article distributed under the Creative Commons Attribution License, which permits unrestricted use, distribution, and reproduction in any medium, provided the original work is properly cited.

The paper addresses the problem of track-to-track association in the presence of sensor biases. In some challenging scenarios, it may be infeasible to implement bias estimation and compensation in time due to the computational intractability or weak observability about sensor biases. In this paper, we introduce the structural feature for each local track, which describes the spatial relationship with its neighboring targets. Although the absolute coordinates of local tracks from the same target are severely different in the presence of sensor biases, their structural features may be similar. As a result, instead of using the absolute kinematic states only, we employee the structural similarity to define the association cost. When there are missed detections, the structural similarity between local tracks is evaluated by solving another $2 \mathrm{D}$ assignment subproblem. Simulation results demonstrated the power of the proposed approach.

\section{Introduction}

The potential advantages of fusing information from disparate sensor systems to achieve better surveillance have been recognized. Track-to-track association $[1,2]$ is a crucial step in the distributed estimation fusion system, which seeks to determine the correspondence between local tracks from different sensors. Unfortunately, sensor reports may be influenced by systematic errors (sensor biases) besides random errors. In this case, sensor registration $[3,4]$ is required to remove the sensor biases from the biased reports. When track association and sensor registration meet each other, there are more challenges. On the one hand, sensor registration is based on the common sensor reports from the same target. On the other hand, the removal of the sensor biases contributes greatly to making correct association assignments. In this sense, sensor registration and track association are tightly coupled together.

An alternative way to tackle this problem is to implement bias estimates and track-to-track association jointly. Several heuristic algorithms, including centroid matching and singleton matching algorithms, for estimating the relative sensor biases in track-to-track association were explored in [5]. Considering the effects of biases, Stone et al. estimated the relative biases based on the fast Fourier transform (FFT) and multiple dimensional spatial cross-correlation function in [6]. In [7], Levedahl modeled this problem as a global nearest pattern (GNP) problem. It produced the bias estimates for each association hypothesis firstly, and the association probability was computed based on the given bias estimates. The final association hypothesis was determined with the maximum probability. In [8], Papageorgiou and Sergi optimized the computation complexity of the GNP algorithm to get the trading-off between the optimality and computation time. In $[9,10]$, Papageorgiou et al. computed the pure association probability by assuming a prior distribution of sensor biases. However, it is hard to get the prior distribution of sensor biases. A joint track association and relative bias estimation problem was formulated and a solution based on the Dijkstra search was developed in [11]. A solution based on the branchand-bound framework for the same problem was described in [12]. In [13], the performance comparisons of several algorithms for estimating relative sensor bias were given. In [14], a feature called "reference topology" based on the relative coordinates is presented. However, its association performance depends on the granularity of cell partitions greatly. Moreover, no systematic method is given to handle the case of missed detections. In $[15,16]$, the joint approaches 
to data association, sensor registration, and track fusion were given by means of the expectation-maximization (EM) algorithm.

In some challenging scenarios, it is impossible in practice to implement bias estimation and compensation in time due to the computational intractability or weak observability about sensor biases. In this paper, we turned to implement track-to-track association without bias estimation. As we know, it is extremely essential to establish a reasonable metric to measure the similarity of local tracks in trackto-track association problem. In general, the similarity of local tracks is based on the absolute kinematic parameters. However, the absolute kinematic parameters are corrupted greatly in the presence of sensor biases. For example, when given the azimuth bias of 3 degrees, the position deviation could reach up to 10 kilometers for a target located $200 \mathrm{~km}$ from the sensor. In this case, the similarity measure based on absolute coordinates is unbelievable anymore. In this paper, we introduce the structural feature for each local track describing the spatial relationship with its neighboring targets. Although the absolute coordinates of local tracks from the same target are severely different in the presence of sensor biases, their structural features may be similar. So, instead of using the absolute kinematic states only, we employ the structural similarity to measure the distance of two local tracks from different sensors.

In this paper, we develop a structural similarity-based approach to deal with the problem of track association in the presence of sensor biases. Main contributions are given as follows. Firstly, under appropriate assumptions, the distance invariance between two targets detected by different sensors is verified in the presence of sensor biases. Secondly, the structural feature is introduced for each local track, which is represented by the distance set from the track to all the other tracks from the same sensor. Thirdly, a two-dimensional (2D) assignment model is established to implement track-to-track association in the presence of sensor biases. Instead of using the absolute kinematic states only, the structural similarity between local tracks is adopted to measure the association cost and is evaluated by solving another $2 \mathrm{D}$ assignment subproblem. Moreover, the dummy track is introduced for each sensor to cope with possibly missed detections. Simulation results demonstrated the power of the proposed approaches.

The rest of the paper is organized as follows. In Section 2 we describe the measurement model and formulate the problem of track-to-track association. Section 3 shows how to implement the track association based on structural similarity. Simulation results are given in Section 4 to demonstrate the effectiveness of the proposed approaches. Finally, concluding remarks and further research directions are in Section 5 .

\section{Problem Formulation}

Consider a multitarget tracking scenario with two sensors and $N_{t}$ targets in the surveillance region. In this paper, false alarms are not considered since they can also be viewed as the targets. In references with the radar sensor which measures the range and azimuth to the target, the measurement process is implemented in the local polar coordinate system (LPCS). Let $z^{m, i} \triangleq\left\{r^{m, i}, \theta^{m, i}\right\}$ be the $i$ th measurement $\left(i=1,2, \ldots, n_{m}\right)$ from sensor $m(m=1,2)$ at the time instant $k$, in which $r^{m, i}$ and $\theta^{m, i}$ mean the range and angle measurement, respectively.

Two kinds of sensor biases are considered in general: range bias and azimuth bias. Since there are systematic biases and random errors, the original measurement $\left\{r^{m, i}, \theta^{m, i}\right\}$ from sensor $m$ can be modeled as

$$
\begin{aligned}
& r^{m, i}=\bar{r}^{m, i}+\Delta r^{m}+v_{r}^{m}, \\
& \theta^{m, i}=\bar{\theta}^{m, i}+\Delta \theta^{m}+v_{\theta}^{m},
\end{aligned}
$$

where $\bar{r}^{m, i}$ and $\bar{\theta}^{m, i}$ denote the real range and azimuth of target $i$ form sensor $m$, respectively. $\Delta r^{m}$ and $\Delta \theta^{m}$ are systematic biases and $v_{r}^{m}$ and $v_{\theta}^{m}$ are random noises. The random noises $\widetilde{V}^{m}=\left[\begin{array}{ll}v_{r}^{m} & v_{\theta}^{m}\end{array}\right]^{T}$ are white Gaussian with zero mean and variances of $\left(\sigma_{r}^{m}\right)^{2}$ and $\left(\sigma_{\theta}^{m}\right)^{2}$, respectively.

Figure 1 depicts a scenario with two sensors and two targets. $\mathbf{T}_{a}\left(\bar{x}^{a}, \bar{y}^{a}\right)$ and $\mathbf{T}_{b}\left(\bar{x}^{b}, \bar{y}^{b}\right)$ are the real positions of targets. $\mathbf{T}_{m, a}\left(x^{m, a}, y^{m, a}\right)$ and $\mathbf{T}_{m, b}\left(x^{m, b}, y^{m, b}\right)$ are the position measurements given by sensor $m(m=1,2)$.

Based on the biased measurements $\left\{r^{m, i}, \theta^{m, i}\right\}$, each sensor $m$ produces its own local tracks represented by two tuples $\left\{\widehat{\mathbf{x}}^{m, i}, \mathbf{P}^{m, i}\right\}\left(m=1,2 ; i=1,2, \ldots, n_{m}\right) . \widehat{\mathbf{x}}^{m, i}$ and $\mathbf{P}^{m, i}$ mean the state estimate and error covariance, respectively. For simplicity, we omit the time index here. Track-to-track association seeks to determine the correspondence between local tracks from different sensors. The key point is to measure the similarity of local tracks. Traditionally, it is defined by the weighted statistical distance under the independence assumption:

$$
\begin{array}{r}
\alpha_{i_{1} i_{2}}=\left(\widehat{\mathbf{x}}^{1, i_{1}}-\widehat{\mathbf{x}}^{2, i_{2}}\right)^{T}\left(\mathbf{P}^{1, i_{1}}+\mathbf{P}^{2, i_{2}}\right)^{-1}\left(\widehat{\mathbf{x}}^{1, i_{1}}-\widehat{\mathbf{x}}^{2, i_{2}}\right) \\
\left(i_{1}=1,2, \ldots, n_{1}, i_{2}=1,2, \ldots, n_{2}\right) .
\end{array}
$$

However, local tracks are biased estimates in the presence of sensor biases. The direct association of biased estimates will not produce a satisfactory result.

\section{Track Association Based on Structural Similarity}

In traditional approaches to track-to-track association, only the absolute kinematic states of targets are considered. In the presence of sensor biases, it does not work well especially for the large azimuth bias, since it may result in severe deviations of absolute coordinates. In this section, the basic principle of the structural similarity is firstly verified, and then the structural similarity is used to measure the distance of local tracks instead of using the absolute kinematic states only. A complete $2 \mathrm{D}$ assignment model to deal with possible missed detections is also addressed.

3.1. Basic Principle of Structural Similarity. In what follows, by assuming small range biases, the distance invariance 


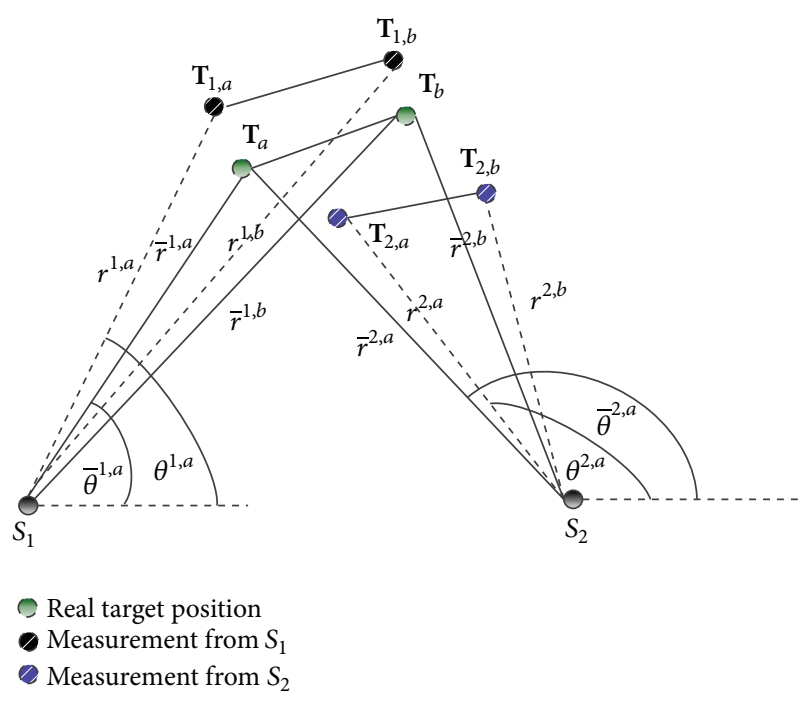

FIgURE 1: Sensor configuration geometry.

between two targets detected by different sensors is verified in the presence of sensor biases. We introduce a structural feature for each local track, which describes the spatial relationship with its neighboring targets. According to the distance invariance, the structural feature of local tracks from the same target is similar. As a result, the structural similarity is employed to define the association cost between local tracks.

The random error $\widetilde{V}^{m}(m=1,2)$ is ignored in the following derivation for the simplicity; thus we have

$$
\begin{aligned}
& r^{m, i}=\bar{r}^{m, i}+\Delta r^{m}, \\
& \theta^{m, i}=\bar{\theta}^{m, i}+\Delta \theta^{m} .
\end{aligned}
$$

In the absence of sensor biases, the real position measurement of the target state is

$$
\left[\begin{array}{l}
\bar{x}^{m, i} \\
\bar{y}^{m, i}
\end{array}\right]=\left[\begin{array}{l}
\bar{r}^{m, i} \cos \left(\bar{\theta}^{m, i}\right) \\
\bar{r}^{m, i} \sin \left(\bar{\theta}^{m, i}\right)
\end{array}\right] .
$$

In the presence of sensor biases, it becomes

$$
\left[\begin{array}{l}
x^{m, i} \\
y^{m, i}
\end{array}\right]=\left[\begin{array}{c}
\left(\bar{r}^{m, i}+\Delta r^{m}\right) \cos \left(\bar{\theta}^{m, i}+\Delta \theta^{m}\right) \\
\left(\bar{r}^{m, i}+\Delta r^{m}\right) \sin \left(\bar{\theta}^{m, i}+\Delta \theta^{m}\right)
\end{array}\right] .
$$

Manipulating the items in the above equations, we have

$$
\begin{aligned}
{\left[\begin{array}{l}
x^{m, i} \\
y^{m, i}
\end{array}\right]=} & \left(\begin{array}{cc}
\cos \Delta \theta^{m} & -\sin \Delta \theta^{m} \\
\sin \Delta \theta^{m} & \cos \Delta \theta^{m}
\end{array}\right)\left[\begin{array}{l}
\bar{x}^{m, i} \\
\bar{y}^{m, i}
\end{array}\right] \\
& +\left(\begin{array}{c}
\Delta r^{m} \cos \left(\theta^{m, i}+\Delta \theta^{m}\right) \\
\Delta r^{m} \sin \left(\theta^{m, i}+\Delta \theta^{m}\right)
\end{array}\right) .
\end{aligned}
$$

From (6), it can be observed that the range bias $\Delta r^{m}$ brings about the translation for targets, and the azimuth bias $\Delta \theta^{m}$ produces the rotation and also contributes to the translation of targets when the range bias is nonzero. Moreover, the azimuth bias is the critical factor compared with the range bias, which produces more severe influence on the target's abstract coordinate and will be enlarged as the distance from the sensor to the target increases.

The real squared distance between two targets $\mathbf{T}_{a}$ and $\mathbf{T}_{b}$ is

$$
d^{2}\left(\mathbf{T}_{a}, \mathbf{T}_{b}\right)=\left(\bar{x}^{a}-\bar{x}^{b}\right)^{2}+\left(\bar{y}^{a}-\bar{y}^{b}\right)^{2} .
$$

For sensor $m(m=1,2)$, the squared distance $d_{m}^{2}\left(\mathbf{T}_{a}, \mathbf{T}_{b}\right)$ between two position measurements $\mathbf{T}_{m, a}$ and $\mathbf{T}_{m, b}$ is

$$
\begin{aligned}
d_{m}^{2}\left(\mathbf{T}_{a}, \mathbf{T}_{b}\right) \triangleq & d^{2}\left(\mathbf{T}_{m, a}, \mathbf{T}_{m, b}\right) \\
= & \left(x^{m, a}-x^{m, b}\right)^{2}+\left(y^{m, a}-y^{m, b}\right)^{2} \\
= & \left(\bar{r}^{m, a}+\Delta r^{m}\right)^{2}+\left(\bar{r}^{m, b}+\Delta r^{m}\right)^{2} \\
& -2\left(\bar{r}^{m, a}+\Delta r^{m}\right)\left(\bar{r}^{m, b}+\Delta r^{m}\right) \\
& \times \cos \left(\theta^{m, a}-\theta^{m, b}\right) .
\end{aligned}
$$

In this way, the difference between $d_{m}^{2}\left(\mathbf{T}_{a}, \mathbf{T}_{b}\right)$ and $d^{2}\left(\mathbf{T}_{a}, \mathbf{T}_{b}\right)$ can be expressed by

$$
\begin{aligned}
D \triangleq & d_{m}^{2}\left(\mathbf{T}_{a}, \mathbf{T}_{b}\right)-d^{2}\left(\mathbf{T}_{a}, \mathbf{T}_{b}\right) \\
= & 2\left(\Delta r^{m}\right)^{2}+2 \Delta r^{m} \bar{r}^{m, a}+2 \Delta r^{m} \bar{r}^{m, b} \\
& -2\left(\left(\Delta r^{m}\right)^{2}+\Delta r^{m} \bar{r}^{m, a}+\Delta r^{m} \bar{r}^{m, b}\right) \\
& \times \cos \left(\bar{\theta}^{m, a}-\bar{\theta}^{m, b}\right) \\
= & 2\left(\left(\Delta r^{m}\right)^{2}+\Delta r^{m} \bar{r}^{m, a}+\Delta r^{m} \bar{r}^{m, b}\right) \\
& \times\left[1-\cos \left(\bar{\theta}^{m, a}-\bar{\theta}^{m, b}\right)\right] .
\end{aligned}
$$

From (9), it is seen that the difference $D$ is only influenced by the range bias $\Delta r^{m}$ and the real position of the target but is independent of azimuth bias $\Delta \theta^{m}$. This reveals that although the azimuth bias may cause a severe impact on the absolute coordinate, it causes less impact on the relative coordinate. When the range bias is too large, the difference $D$ may be severe. Fortunately, the range bias in practical applications is generally at a low level. That means that the distance between two targets detected by different sensors differs slightly, which is less sensitive to sensor biases. In what follows, we employ the structural similarity to measure the distance between two local tracks from different sensors.

3.2. 2D Assignment Model for Track Association. As we know, it is very important to establish a reasonable metric to measure the similarity of local tracks from different sensors. This problem becomes extremely complicated in the presence of sensor bias, random errors, and missed tracks. Figure 2 


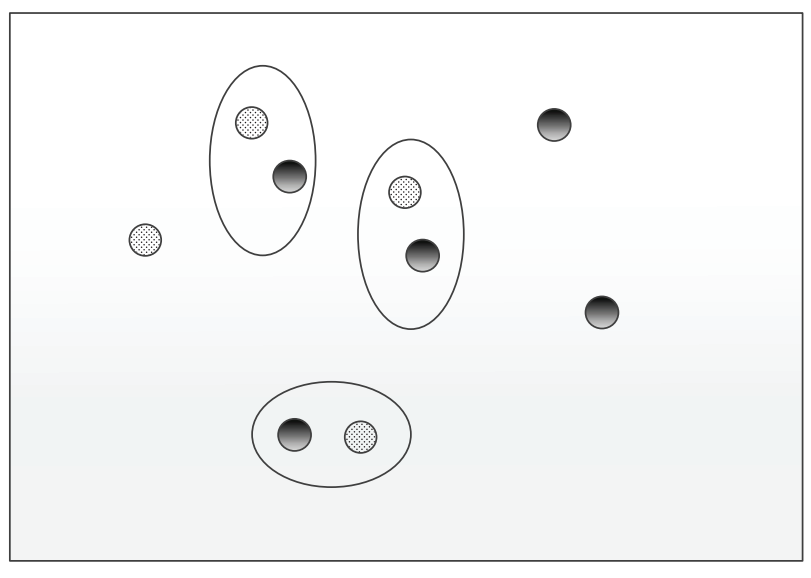

Measurement from sensor 1

Measurement from sensor 2

In which measurements from the same target

FIGURE 2: Distribution of measurements from different sensors.
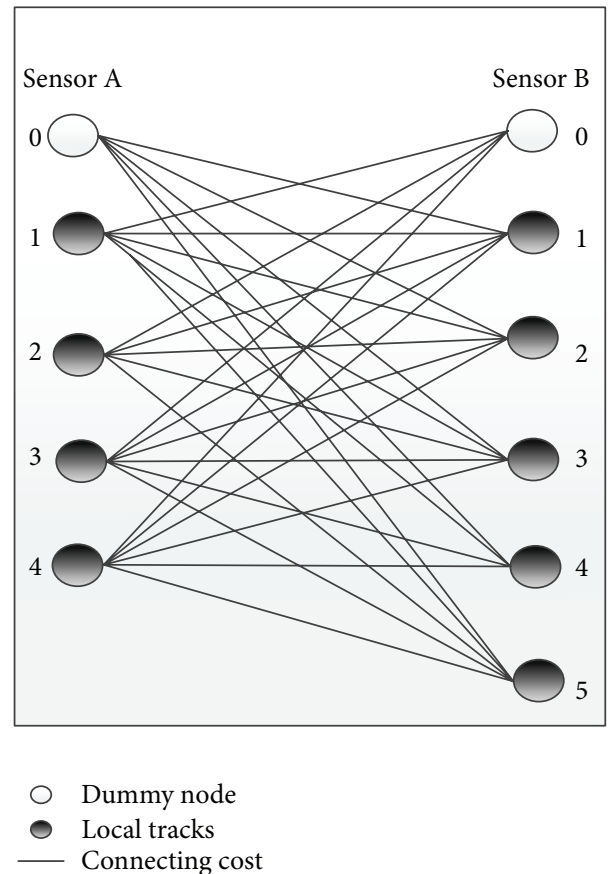

FIGURE 3: Graphical representation of track association problem.

illustrates a scenario with two sensors and six targets. Here, sensor 1 detects 4 targets, and sensor 2 detects 5 targets. That means that $n_{1}=4, n_{2}=5$, and there are 3 common targets.

For the scenario in Figure 2, a graphical representation of track association is shown in Figure 3. We introduce a node for each local track. To deal with possible missed detections, a dummy node is introduced for each local sensor which provides the access to link an isolated track with the dummy track. The edges mean possible links between different local tracks.
In the following, a 2D assignment model with given constraints is established by minimizing the sum of all pairwise costs:

$$
\begin{array}{ll}
\min & \sum_{i_{1}=0}^{n_{1}} \sum_{i_{2}=0}^{n_{2}} c_{i_{1} i_{2}} \rho_{i_{1} i_{2}} \\
\text { s.t } \quad \sum_{i_{2}=0}^{n_{2}} \rho_{i_{1} i_{2}}=1, \quad \forall i_{1}=1,2, \ldots, n_{1} \\
\sum_{i_{1}=0}^{n_{1}} \rho_{i_{1} i_{2}}=1, \quad \forall i_{2}=1,2, \ldots, n_{2} \\
\rho_{i_{1} i_{2}} \in\{0,1\}, \quad \forall i_{1}=0,1,2, \ldots, n_{1}, \\
\forall i_{2}=0,1,2, \ldots, n_{2} .
\end{array}
$$

3.3. Association Cost of Local Nodes Based on the Structural Similarity. The model (10) can be solved efficiently and optimally by the general $2 \mathrm{D}$ assignment algorithms, such as auction algorithm and Hungarian algorithm. Now, the remaining problem in solving (10) is how to define the connecting $\operatorname{cost} c_{i_{1} i_{2}}$. Instead of using the absolute kinematic states only, the structural similarity of local tracks is employed here. Three kinds of costs need to be specified: between local tracks, between dummy tracks, and between local track and the dummy track.

Firstly, we define a structural feature for each node $N_{i_{m}}$ $\left(m=1,2 ; i_{m}=1, \ldots, n_{m}\right)$, which is represented by the set $I_{i_{m}}$ including all the distances from local track $i_{m}$ to $j(j=$ $\left.1,2, \ldots, n_{m}, j \neq i_{m}\right)$; that is,

$$
I_{i_{m}}=\left\{d\left(j, i_{m}\right)\right\}_{j=1,2, \ldots, n_{m}, j \neq i_{m}}
$$

We discuss the definition of $c_{i_{1} i_{2}}$ in terms of the following three cases.

(1) With Unity Detection Probability $P_{D_{m}}\left(P_{D_{m}}=1, m=1,2\right)$. If $P_{D_{m}}=1(m=1,2)$, then $n_{1}=n_{2}=n$. We order the elements of set $I_{i_{m}}$ from the smallest to largest: $\widetilde{d}_{i_{m}, 1} \leq \widetilde{d}_{i_{m}, 2} \leq$ $\cdots \leq \widetilde{d}_{i_{m}, n-1}$. In this way, the cost $c_{i_{1} i_{2}}$ (for all $i_{1}=1,2, \ldots, n_{1}$, for all $i_{2}=1,2, \ldots, n_{2}$ ) can be defined by

$$
c_{i_{1} i_{2}}=D\left(I_{i_{1}}, I_{i_{2}}\right)=\sum_{j=1}^{n-1}\left|\widetilde{d}_{i_{1}, j}-\widetilde{d}_{i_{2}, j}\right| .
$$

(2) Without Unity Detection Probability $P_{D_{m}}\left(P_{D_{1}} \cdot P_{D_{2}} \neq 1\right)$. If $P_{D_{m}}<1(m=1,2)$, the computation of $c_{i_{1} i_{2}}$ (for all $i_{1}=$ $1,2, \ldots, n_{1}$, for all $\left.i_{2}=1,2, \ldots, n_{2}\right)$ becomes complicated due to possible missed detections. To define the connection cost $c_{i_{1} i_{2}}$, a $2 \mathrm{D}$ assignment subproblem (13) is constructed. As shown in Figure 4, a graphical representation is established to compute the cost $c_{23}$. As done in the optimization model 


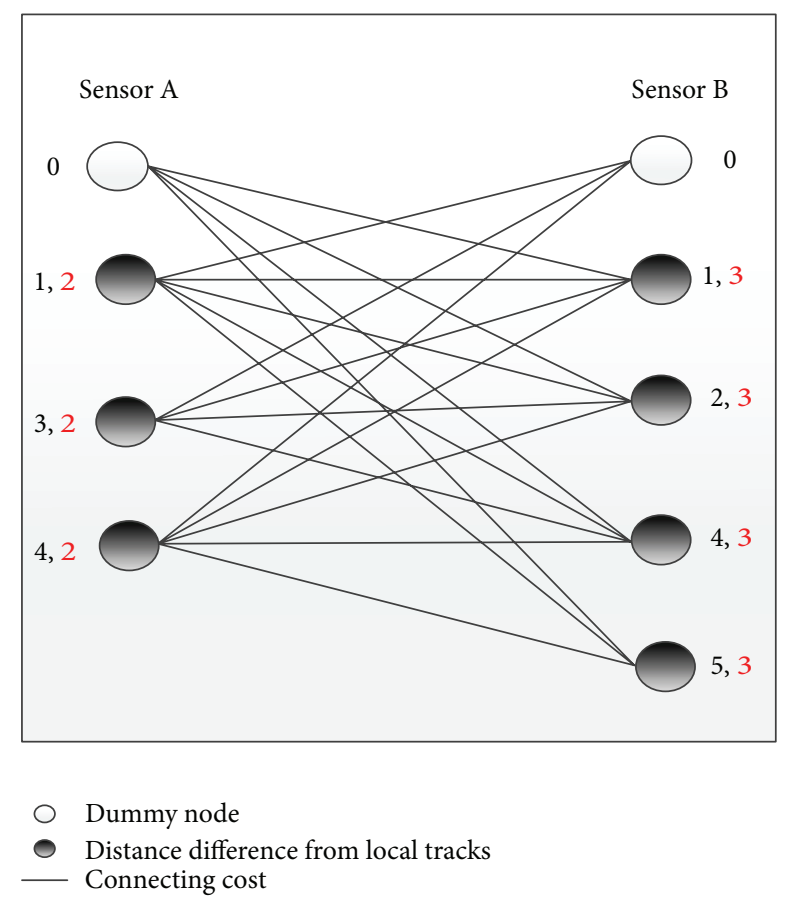

FIGURE 4: Graphical representation of 2D assignment subproblem to compute the cost $c_{23}$.

(10), two dummy nodes are introduced to cope with the case of missed detections:

$$
\begin{aligned}
& J_{i_{1} i_{2}}=\min \quad \sum_{j_{1}=0, j_{1} \neq i_{1}}^{n_{1}} \sum_{j_{2}=0, j_{2} \neq i_{2}}^{n_{2}} \widetilde{\rho}_{j_{1} j_{2}} \tilde{\rho}_{j_{1} j_{2}} \\
& \text { s.t } \quad \sum_{j_{2}=0, j_{2} \neq i_{2}}^{n_{2}} \widetilde{\rho}_{j_{1} j_{2}}=1, \quad \forall j_{1}=1,2, \ldots, n_{1}, j_{1} \neq i_{1} \\
& \sum_{j_{1}=0, j_{1} \neq i_{1}}^{n_{1}} \widetilde{\rho}_{j_{1} j_{2}}=1, \quad \forall j_{2}=1,2, \ldots, n_{2}, j_{2} \neq i_{2} \\
& \widetilde{\rho}_{j_{1} j_{2}} \in\{0,1\}, \quad \forall j_{1}=0,1,2, \ldots, n_{1}, \\
& \forall j_{2}=0,1,2, \ldots, n_{2}, \quad j_{1} \neq i_{1}, \quad j_{2} \neq i_{2} .
\end{aligned}
$$

In this optimization model (13), the critical part is to define the cost $\widetilde{c}_{j_{1} j_{2}}$. For all $j_{1}=1,2, \ldots, n_{1}, j_{1} \neq i_{1}$, and for all $j_{2}=1,2, \ldots, n_{2}, j_{2} \neq i_{2}$, the cost $\widetilde{c}_{j_{1} j_{2}}$ is defined by the absolute value of distance difference:

$$
\widetilde{c}_{j_{1} j_{2}}=\left|d\left(j_{1}, i_{1}\right)-d\left(j_{2}, i_{2}\right)\right|
$$

If track-pair $\left\{i_{1}, j_{1}\right\}$ from sensor 1 and $\left\{i_{2}, j_{2}\right\}$ from sensor 2 share the same two targets, then $\widetilde{c}_{j_{1} j_{2}}$ would be a small quantity due to the structural similarity. When given a threshold $\lambda$, we compare $\widetilde{c}_{j_{1} j_{2}}$ and the gate value $\lambda$. If $\widetilde{c}_{j_{1} j_{2}}>\lambda$, then it means that track-pair $\left\{i_{1}, j_{1}\right\}$ from sensor 1 and $\left\{i_{2}, j_{2}\right\}$ from sensor 2 do not share the same targets. To prevent them from associating, we set $\widetilde{c}_{j_{1} j_{2}}=+\infty$. In practical operations, we set $\widetilde{c}_{j_{1} j_{2}}=\Gamma$, where $\Gamma$ is a large positive integer. In addition, we set $\widetilde{c}_{0 j_{2}}=\widetilde{c}_{j_{1} 0}=10^{-2} \Gamma$ to encourage the isolated node to link with the dummy node. In addition, we set $\widetilde{\mathcal{c}}_{00}=\Gamma$.

As far, a complete 2D assignment subproblem (13) is well defined. By solving it, the optimal fitness $J_{i_{1} i_{2}}^{*}$ can be obtained. It is noted that $c_{i_{1} i_{2}}$ cannot be defined directly by $J_{i_{1} i_{2}}^{*}$; further processing is required considering possible isolated tracks. We denote the number of valid associations by $\beta$, which is defined as the number of association assignments between local tracks (not with dummy nodes). If $\beta$ is close enough to the expected number $\alpha$ of common targets $(|\beta-\alpha| \leq \varepsilon$, $\varepsilon$ is a small positive integer), then $c_{i_{1} i_{2}}=J_{i_{1} i_{2}}^{*}$. Otherwise, set $c_{i_{1} i_{2}}=\Gamma$, where the expected number $\alpha$ of common targets is computed by

$$
\alpha=\frac{P_{D_{1}} P_{D_{2}}\left(n_{1}+n_{2}\right)}{\left(1+P_{D_{1}} P_{D_{2}}\right)} .
$$

It is proportional to the detection probability $P_{D_{m}}(m=1,2)$ of sensors. If $P_{D_{m}}=1(m=1,2)$, then $\alpha=n_{1}=n_{2}$.

(3) Connection Cost with Dummy Node. When a local node $\left(i_{1}\right.$ or $\left.i_{2}\right)$ is declared isolated, we set $c_{0 i_{2}}=c_{i_{1} 0}=10^{-2} \Gamma$ to encourage it to link with the dummy node. In addition, we set $c_{00}=\Gamma$ to discourage the association between dummy tracks.

\subsection{Discussions}

(1) Approximation Solution Based on the Nearest Neighborhood Method. A new approach to track-to-track association in the presence of sensor biases is given in Sections 3.2 and 3.3, which requires solving two $2 \mathrm{D}$ optimal assignment models. One serves to compute the connecting cost between two local tracks, and the other one is to determine the final association assignment between two sets of local tracks. To simplify, we call this method "structural similarity-based 2D assignment" (SS-based 2D assignment). Considering the complexity of the proposed model, an approximation solution can be obtained by using the nearest neighborhood (NN) method when determining the final association assignment, instead of using the optimal assignment. Such a scheme is denoted by structural similarity-based nearest neighborhood (SS-based $\mathrm{NN}$ ). Simulation results provided before indicate that such an approximate method can also provide a better association result, because the structural property is considered when constructing the connection cost between local tracks.

(2) Possible Extensions. In practical fusion applications, heterogeneous sensors are commonly found. The proposed approach can also work well in this case, since what we handle here are local tracks after local processing. Although the measuring process is implemented in the local measurement coordinate system, local tracks from heterogeneous sensors may be in the common Cartesian coordinate system.

In addition, in short-range applications or for maritime surveillance, different scattering centres of the objects under consideration may give rise to several distinct detections. At this time, what one is concerned with includes the state 
for both the target kinematics and the target extension. The proposed approach fails to deal with the case that the targets have more than one scattering centers. Readers may refer to literatures concerning extended target tracking $[17,18]$. It is still a problem which few people get into to implement trackto-track association between extended targets.

\section{Simulation Results}

4.1. Scenario Setup and Results. In this section, we provide some simulation results to illustrate the efficiencies of the proposed approaches ("SS-based 2D assignment" and "SSbased NN"). Here, we consider a multiple target tracking scenario with two sensors. At the beginning of the tracking process, 30 targets are distributed uniformly in the region $[20 \mathrm{~km}, 40 \mathrm{~km}] \times[20 \mathrm{~km}, 40 \mathrm{~km}]$. The motion of all the targets follows the constant acceleration (CA) model with acceleration $1 \mathrm{~m} / \mathrm{s}^{2}$. The process noise is assumed white Gaussian with zero mean and covariance matrix $Q=\left(0.1 \mathrm{~m} / \mathrm{s}^{3}\right) \mathbf{I}_{2}$, where $\mathbf{I}_{2}$ is an $2 \times 2$ identity matrix. The initial direction of velocity occurs randomly in $[0,2 \pi]$ with the amplitude $100 \mathrm{~m} / \mathrm{s}$. The sample interval $T$ is set to $1 \mathrm{~s}$, and the tracking time is $30 \mathrm{~s}$. Two sensors are located at $(40000 \mathrm{~m}, 5000 \mathrm{~m})$ and $(15000 \mathrm{~m}$, $10000 \mathrm{~m}$ ) in the global Cartesian coordinate system (GCCS), respectively. Each sensor measures the range and angle to the target. The random range error for both sensors is modelled as white Gaussian noises with the standard deviations $\sigma_{r}^{1}=$ $30 \mathrm{~m}$ and $\sigma_{r}^{2}=40 \mathrm{~m}$, respectively. The random angle error is also modelled as white Gaussian noises with standard deviations $\sigma_{\theta}^{1}=0.2^{\circ}$ and $\sigma_{\theta}^{2}=0.1^{\circ}$. Both of the sensors employ EKF to obtain the local tracks based on their own measurement information. The measurements are supported by 50 MonteCarlo runs performed on the same target trajectories but with independently generated measurements for each trial. Except for the special declaration in the following simulations, the detection probabilities of the two sensors are $P_{d_{1}}=0.8$ and $P_{d_{2}}=0.7$; the systematic biases are set to $\Delta r_{1}=400 \mathrm{~m}$, $\Delta r_{2}=500 \mathrm{~m}, \Delta \theta_{1}=6^{\circ}, \Delta \theta_{2}=-5^{\circ}$.

Performance comparisons are made between the proposed approaches and the competing algorithms. In competing algorithms, the square Mahalanobis distance is chosen as the similarity measure of two local tracks. The final association assignment is determined based on the $2 \mathrm{D}$ assignment algorithm and the nearest neighborhood (NN) algorithm, respectively. To simplify, they are called "bias-ignorant $2 \mathrm{D}$ assignment" and "bias-ignorant NN."

We adopt the probability of correct association $P_{c}(t)$ at the time $t$ to evaluate the association performance:

$$
P_{c}(t)=\frac{\sum_{i=1}^{L} C^{i}(t)}{L \times N},
$$

where $C^{i}(t)$ denotes the number of tracks with correct association at time $t$ at the $i$ th Monte-Carlo run, $L$ is the total number of Monte-Carlo runs, and $N$ is the number of targets.

The average probability of correct association $A P_{c}$ is computed by

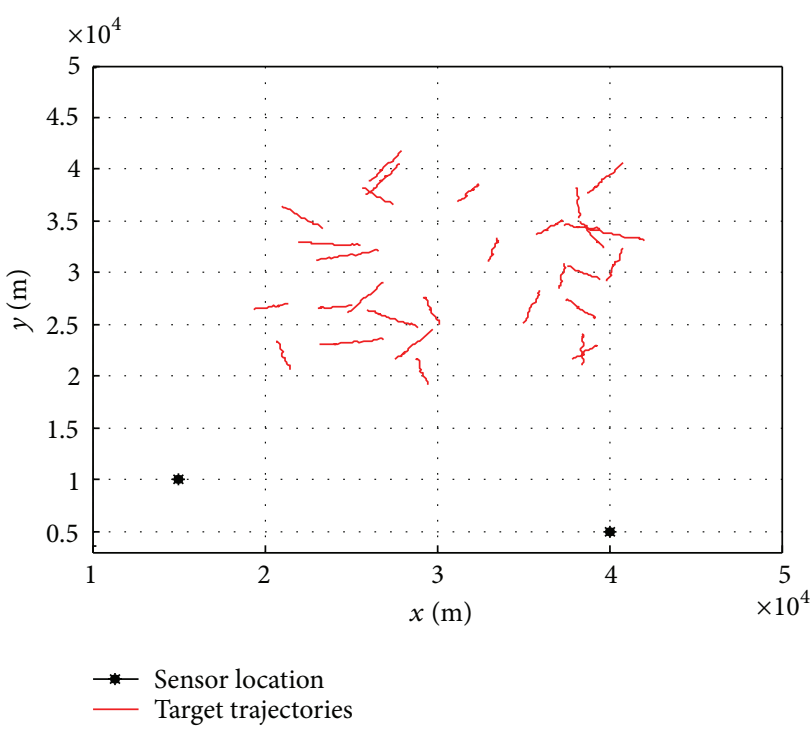

FIGURE 5: Sensors and target trajectories.

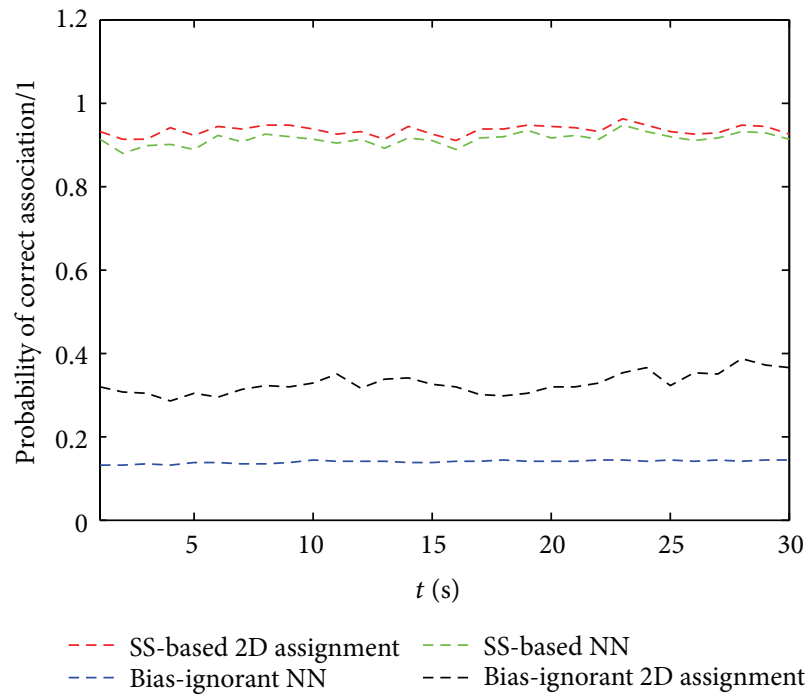

FIgURE 6: Probability of correct association via the whole tracking time.

$$
A P_{c}=\frac{\sum_{i=1}^{K} P_{c}(t)}{K}
$$

where $K$ is the overall tracking time.

Figure 5 shows sensor locations and target trajectories during the whole tracking time in one run. The probability comparison of the correct association via the tracking time is shown in Figure 6.

Figures 7-10 illustrate the average probabilities of correct association via different detection probability, angle bias, range bias, and the total number of targets, respectively. In Figure 7, the detection probability $P_{D_{1}}$ of sensor 1 is set to 0.8 , and the detection probability $P_{D_{2}}$ of sensor 2 varies from 0.5 to 1 . In Figure 8 , the angle bias $\Delta \theta_{1}$ of sensor 1 is $1^{\circ}$, and the angle bias $\Delta \theta_{2}$ of sensor 2 varies from $0^{\circ}$ to $7^{\circ}$. In Figure 9, 


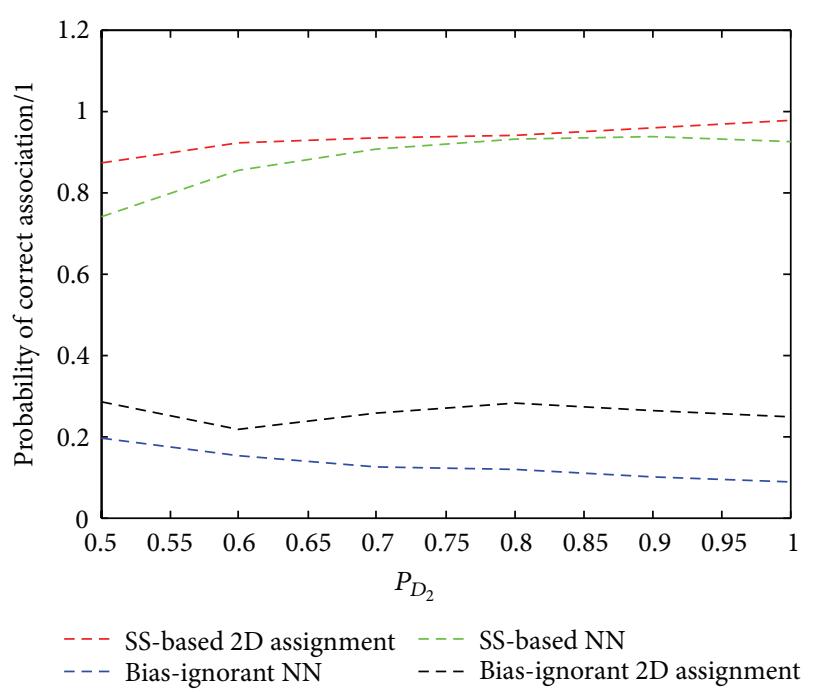

FiguRE 7: Average probability of correct association via different detection probabilities.

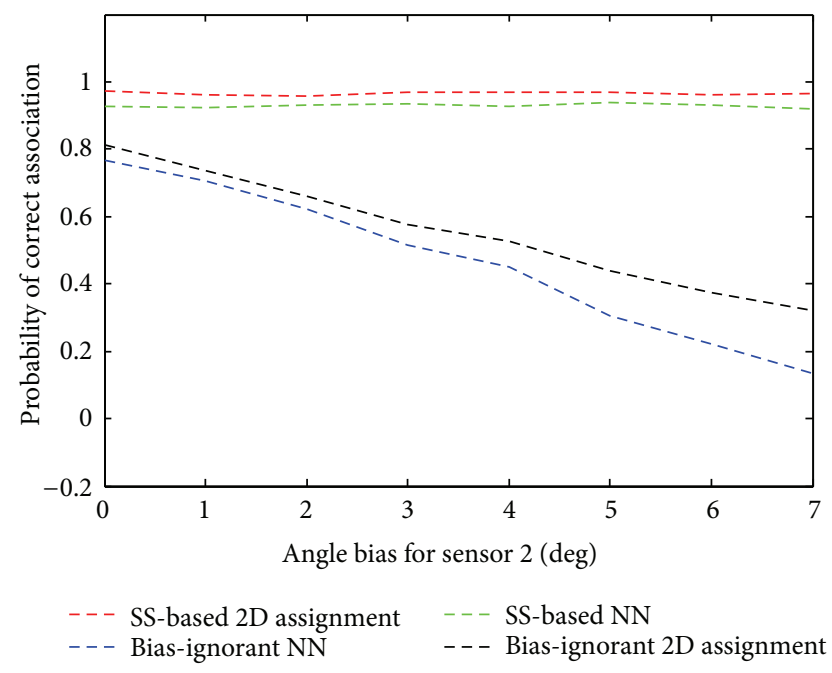

Figure 8: Average probability of correct association via different angle biases.

the range bias of sensor $1 \Delta r_{1}$ varies from $0 \mathrm{~m}$ to $1000 \mathrm{~m}$, and the one of sensor $2 \Delta r_{2}$ is $500 \mathrm{~m}$. In Figure 10, the total number of targets varies from 10 to 70 .

4.2. Result Analysis. From Figures 6-10, it is shown that the proposed "SS-based 2D assignment" and "SS-based NN" outperform the competing algorithms. Moreover, "SS-based NN" is inferior to "SS-based 2D assignment" just a little bit due to the fact that the structural property is considered when constructing the association cost. From Figure 8, it is seen that, as the angle bias increases, the association performances of the "bias-ignorant 2D assignment" and "bias-ignorant NN" experience severe degradations. However, the proposed approaches still perform well, which are insensitive to the angle bias. In addition, it is observed from Figure 10 that the probabilities of correct association given by all methods

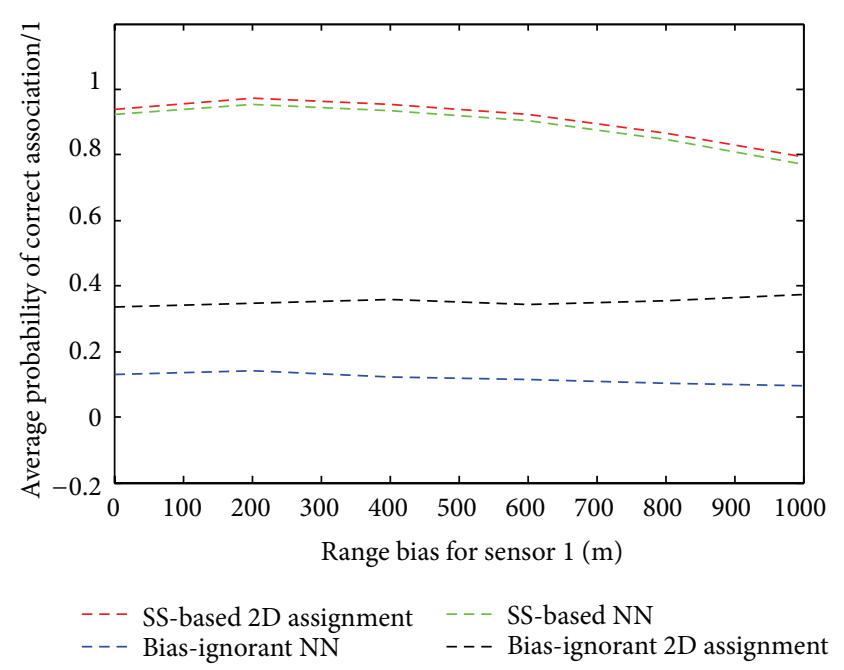

FIGURE 9: Average probability of correct association via different range biases.

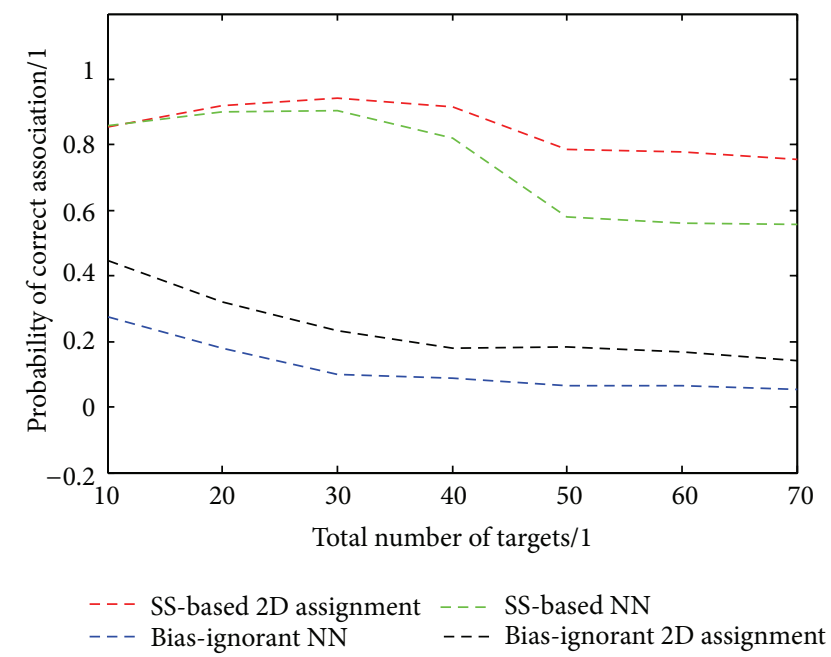

FIGURE 10: Average probability of correct association via the total number of targets.

decrease to some extent as the total number of targets becomes large.

Although the proposed approach performs well in the simulation experiments, it has some flaws. Firstly, the range bias is assumed to be a small one when using the proposed approach; otherwise, the distance invariance between two targets detected by different sensors cannot hold. Secondly, it is required to solve a $2 \mathrm{D}$ assignment model when computing the association cost. Doing so may result in a high computational burden, especially for a large number of targets.

\section{Conclusions}

The sensor reports are disturbed by not only random errors, but also sensor biases. When track-to-track association and sensor registration meet each other, some challenging problems arise since they are tightly coupled together. In this 
paper, instead of using the absolute kinematic states only, the structural features of local tracks are employed for track-totrack association in the presence of sensor biases. A complete $2 \mathrm{D}$ assignment model for track-to-track association is given to deal with possibly missed detections. Lots of simulation results demonstrated the power of the proposed approaches. Further work includes the utilization of multiscan information and the extension of the proposed approach to the multisensor case.

\section{Conflict of Interests}

The authors declare that there is no conflict of interests regarding the publication of this paper.

\section{Acknowledgments}

This research is sponsored by the National Natural Science Foundation of China (61203220/F030307) and State Key Program for Basic Research of China (2013CB329405).

\section{References}

[1] L. M. Kaplan, Y. Bar-Shalom, and W. D. Blair, "Assignment costs for multiple sensor track-to-track association," IEEE Transactions on Aerospace and Electronic Systems, vol. 44, no. 2, pp. 655-677, 2008.

[2] Y. Bar-Shalom and H. Chen, "Multisensor track-to-track association for tracks with dependent errors," Jounal of Advances in Information Fusion, vol. 1, no. 1, pp. 3-14, 2006.

[3] N. Okello and B. Ristic, "Maximum likelihood registration for multiple dissimilar sensors," IEEE Transactions on Aerospace and Electronic Systems, vol. 39, no. 3, pp. 1074-1083, 2003.

[4] X. Lin, Y. Bar-Shalom, and T. Kirubarajan, "Exact multisensor dynamic bias estimation with local tracks," IEEE Transactions on Aerospace and Electronic Systems, vol. 40, no. 2, pp. 576-590, 2004.

[5] S. Mori and Y. Chong, "Effects of unpaired objects and sensor biases on track-to-track association: problems and solutions," in in Proceedings of MSS National Symposium on Sensor and Data Fusion, vol. 1, pp. 137-151, San Antonio, Tex, USA, June 2000.

[6] L. D. Stone, M. L. Williams, and T. M. Tran, "Track-to-track association and bias removal," in Signal and data Processing of Small Targets, Proceedings of SPIE, pp. 315-329, Orlando, Fla, USA, April 2002.

[7] M. Levedahl, "An explicit pattern matching assignment algorithm," in Signal and data Processing of Small Targets, Proceedings of SPIE, pp. 461-469, Orlando, Fla, USA, April 2002.

[8] D. J. Papageorgiou and J.-D. Sergi, "Simultaneous track-to-track association and bias removal using multistart local search," in Proceedings of the IEEE Aerospace Conference (AC '08), March 2008.

[9] D. J. Papageorgiou and M. Holender, “Track-to-track association and ambiguity management in the presence of sensor bias," in Proceedings of the 12th International Conference on Information Fusion (FUSION '09), pp. 2012-2019, Seattle, Wash, USA, July 2009.

[10] J. P. Ferry, "Exact bias removal for the track-to-track association problem," in Proceedings of the 12th International Conference on
Information Fusion (FUSION '09), pp. 1642-1649, Seattle, Wash, USA, July 2009.

[11] M. Levedahl, "Method and System for Assigning Observations," United States Patent US, 7092924 B1, 2006.

[12] S. Danford, S. Herman, B. Kragel, and A. Poore, "A branch and bound framework for joint MAP bias estimation and data association," in Proceedings of the Workshop on Radar Resolution, Nonlinear Estimation, and the Gratuitous Remarks on the Back of Envelope: A Tribute to Fred Daum, Monterey, Calif, USA, May 2007.

[13] S. Mori and C. Chong, "Comparison of bias removal algorithms in track-to-track association," in Signal and Data Processing of Small Targets, vol. 6699 of Proceedings of SPIE, pp. 1-9, San Diego, Calif, USA, August 2007.

[14] X. Du, Y. Wang, and X. Shan, "Track-to-track association using reference topology in the presence of sensor bias," in Proceedings of the 10th International Conference on Signal Processing (ICSP '10), pp. 2196-2201, Beijing, China, October 2010.

[15] Z. Li, S. Chen, H. Leung, and É. Bossé, "Joint data association, registration, and fusion using EM-KF," IEEE Transactions on Aerospace and Electronic Systems, vol. 46, no. 2, pp. 496-507, 2010.

[16] D. L. Huang, H. Leung, and E. Bosse, "A pseudo-measurement approach to simultaneous registration and track fusion," IEEE Transactions on Aerospace and Electronic Systems, vol. 48, no. 3, pp. 2315-2331, 2012.

[17] J. W. Koch, "Bayesian approach to extended object and cluster tracking using random matrices," IEEE Transactions on Aerospace and Electronic Systems, vol. 44, no. 3, pp. 1042-1059, 2008.

[18] K. Granström and U. Orguner, "A PHD filter for tracking multiple extended targets using random matrices," IEEE Transactions on Signal Processing, vol. 60, no. 11, pp. 5657-5671, 2012. 


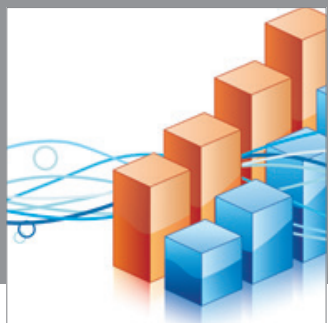

Advances in

Operations Research

mansans

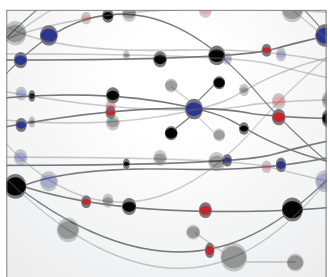

The Scientific World Journal
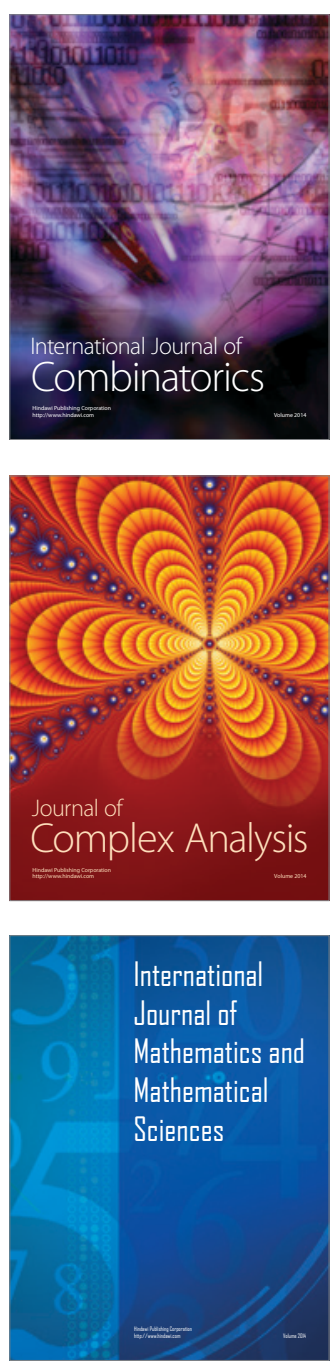
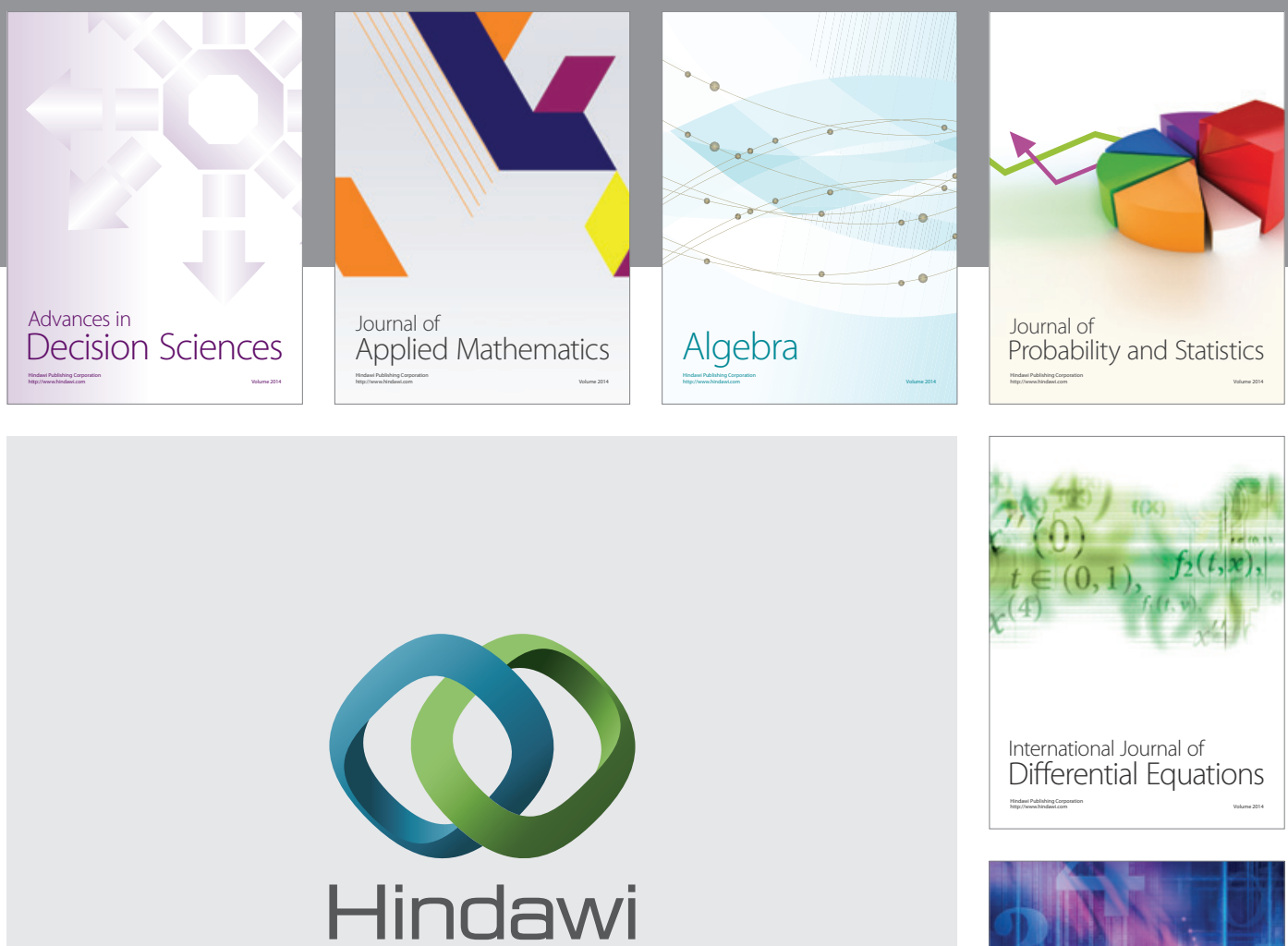

Submit your manuscripts at http://www.hindawi.com
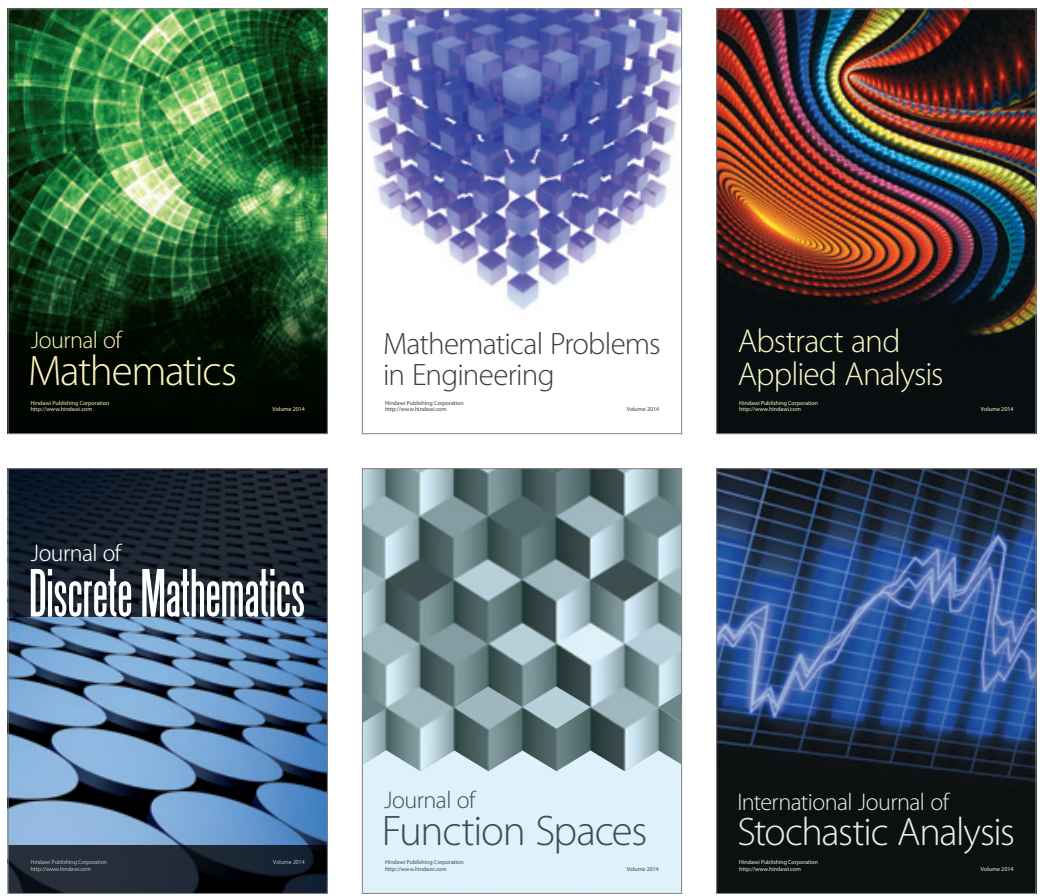

Journal of

Function Spaces

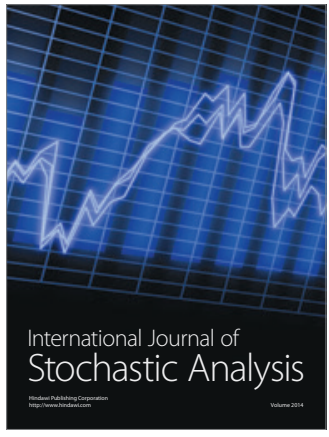

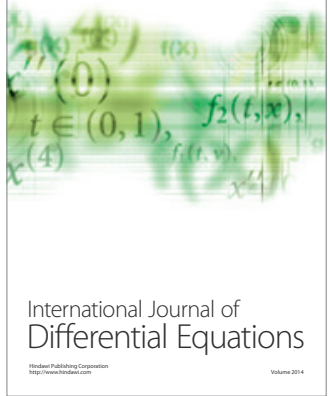
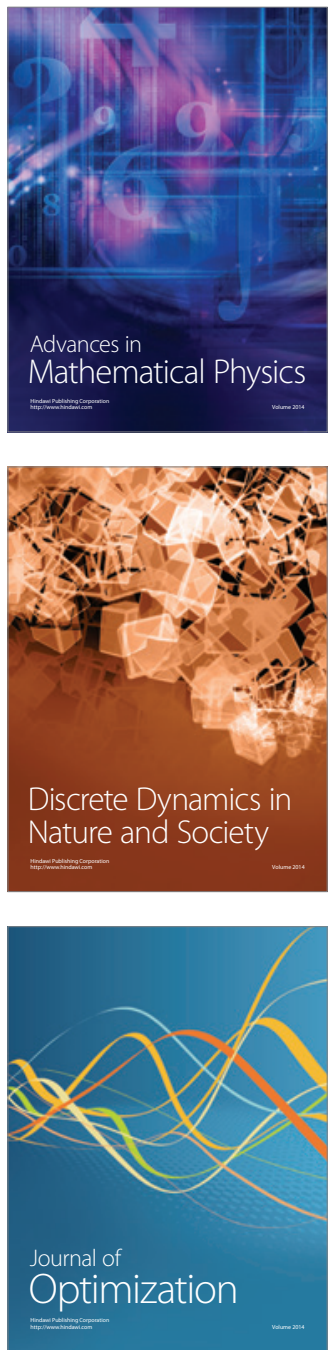